\title{
Valley-Hall topological plasmons in a graphene nanohole plasmonic crystal waveguide
}

\author{
Jian Wei You, Member, IEEE, Zhihao Lan, Qiaoliang Bao, and Nicolae C. Panoiu, Member, IEEE
}

\begin{abstract}
We demonstrate that unidirectional backscatteringimmune propagation of terahertz optical waves can be achieved in a topological valley-Hall waveguide made of graphene nanohole plasmonic crystals. In order to gain deeper physical insights into these phenomena, the band diagram of graphene nanohole plamsonic crystals has been investigated and optimized. We found that a graphene plasmonic crystal with nanohole arrays belonging to the $C_{6 v}$ symmetry group possesses gapless Dirac cones, which can be gapped out by introducing extra nanoholes such that the symmetry point group of the system is reduced from $C_{6 v}$ to $C_{3 v}$. Taking advantage of this feature, we design a mirror symmetric domain-wall interface by placing together two optimized graphene plasmonic crystals so as to construct valleypolarized topological interface modes inside the opened bandgap. Our computational analysis shows that the valley-Hall topological domain-wall interface modes can be achieved at an extremely deep subwavelength scale, and do not rely on the application of external static magnetic fields. This work may pave a new way to develop highly-integrated and robust terahertz plasmonic waveguides at deep-subwavelength scale.
\end{abstract}

Index Terms-Topological plasmon mode propagation, chirality-momentum locking, graphene plasmonic crystal waveguides, valley-Hall topological waveguides, domain-wall interface.

\section{INTRODUCTION}

$\mathbf{T}$ OPOLOGICAL photonics has recently attracted a great deal of attention, chiefly due to the unique opportunities it provides to manipulate light in a robust way, immune to structural imperfections and backscattering [1]. In [2], an analogue of the quantum Hall edge states in photonic crystals containing time-reversal-breaking magneto-optical media was proposed theoretically for the first time. Soon afterwards, such topological edge modes were observed directly in microwave experiments [3]. Since then, the photonic topological states have been extensively studied both from a fundamental science viewpoint [4]-[7], as well as from the perspective of potential applications [8]-[14]. For instance, a number of topological photonic waveguides [8]-[10] have been developed to reduce

Manuscript received xxx, 2020. This work was supported by the European Research Council (ERC), Grant Agreement no. ERC-2014-CoG-648328. (Corresponding author: Nicolae C. Panoiu.)

J. W. You, Z. Lan, and N. C. Panoiu are with the Department of Electronic and Electrical Engineering, University College London, London, WC1E7JE, United Kingdom. (e-mail: j.you@ucl.ac.uk, z.lan@ucl.ac.uk, and n.panoiu@ucl.ac.uk)

Q. Bao is with the Department of Materials Science and Engineering, and Australian Research Council Centre of Excellence in Future LowEnergy Electronics Technologies, Monash University, Clayton, Victoria 3800, Australia. (e-mail: qiaoliang.bao@monash.edu)

Color versions of one or more of the figures in this paper are available online at http://ieeexplore.iee.org.

Digital Object Identifier 10.1109/JSTQE.2020.XXXX. the back-reflection in the presence of inherent structural disorder. In addition, some other topological photonic devices, such as robust delay lines [11], [12] and topological lasers [13], [14], have also been proposed and demonstrated experimentally.

One of the prerequisites of the development of efficient onchip photonic integrated circuits at the nanoscale, is achieving a tight optical field confinement beyond the diffraction limit of light. Graphene [15]-[17] has been shown to be a promising platform to facilitate reaching this goal, due to the fact that its physical properties can be easily controlled by voltage-tuning or chemical doping. Moreover, graphene plasmons, the energy carriers in graphene based photonic systems, exhibit a tight optical field confinement and have long intrinsic relaxation times in the terahertz and up to mid-infrared frequency range [18]-[22]. Indeed, it has been shown that a periodically patterned graphene nanostructure under a static magnetic field can host topological one-way edge plasmons up to infrared frequencies [23]-[25], owing to the breaking of the time reversal symmetry.

Topological transport of plasmons in graphene nanostructures can also be realized in a time reversal symmetric manner by exploiting the idea of valley-contrast transport, via electrically biased patterned metagates [26] or inversion symmetry broken honeycomb lattices of periodically arranged graphene nanodisks [27], [28]. Recently, a large area of periodic graphene nanohole arrays with a period down to $100 \mathrm{~nm}$ has been fabricated [29]-[31]. Compared to the crystals of periodically arranged graphene nanodisks, graphene nanohole crystals offer the particularly useful feature of being an electrically connected surface, yet the band structure of the graphene plasmons can be easily engineered in a purely geometric manner [32]. To the best of our knowledge, topological plasmons in graphene nanohole crystals have not been achieved in the absence of a static magnetic field.

In this work, we propose a novel valley-Hall topological graphene plasmonic crystal waveguide comprised of periodically patterned air nanoholes. To achieve this goal, we firstly construct an inversion-symmetry protected Dirac cone by creating a $C_{6 v}$-symmetric array of nanoholes into a graphene sheet. Subsequently, in order to break the spatial inversion symmetry, we introduce additional nanoholes with different radius into this graphene nanohole crystal. In this way, the gapless Dirac cone is gapped out and consequently a topological bandgap emerges. In order to study the topological features of this novel graphene nanohole crystal containing two kinds of nanoholes, the radius of the additional set of nanoholes is optimized so as to maximize the width of 
the emerging topological bandgap. Taking advantage of this wide topological bandgap, we place two optimized graphene nanohole crystals together in a mirror symmetric manner, so as to construct a domain-wall interface, which gives rise to a valley-Hall plasmonic edge mode inside the topological bandgap.

Employing comprehensive numerical simulations, the valley-Hall plasmon propagation along this carefully-designed interface has been studied, and some typical topological propagation features have been observed, including unidirectional backscattering-immune propagation. Moreover, we have explored the influence of the intrinsic loss of graphene on the characteristic loss propagation length of valley-Hall topological plasmons in this graphene nanohole crystal waveguide, too, and found out that the plasmon lifetime is a key parameter in determining the plasmon propagation performance. Compared to previously studied topological magneto-plasmons, the proposed valley-Hall topological plasmons investigated in this work are time-reversal-invariant and do not depend on external static magnetic fields. Therefore, our proposal would greatly simplify experimental implementations of topological graphene plasmons and could be extremely useful for the development of robustly integrated on-chip photonic devices.

\section{THE AIM OF THE STUDY AND ITS KEY IDEAS}

The aim of this study is to design a topological waveguide for terahertz plasmonic waves based on quantum valley-Hall like effects using graphene nanohole plasmonic crystals (see the illustration in Fig. 1). The waveguide consists of a domainwall interface, which is highlighted in Fig. 1 by a transparent green strip along the $x$-axis, and it is constructed by joining together two properly-designed graphene nanohole crystals referred to as domains $D 1$ and $D 2$. The $D 1$ domain is the mirror symmetric counterpart of the $D 2$ domain, with respect to a plane passing through the domain-wall interface and perpendicular onto the $y$-axis. The unit cells of the $D 1$ and $D 2$ domains are marked by blue and red hexagons, respectively. Each hexagonal unit cell consists of three small nanoholes and three large nanoholes etched in a graphene sheet and the unit cells are arranged periodically in a hexagonal lattice. The array of large nanoholes has $C_{6 v}$ symmetry and in the absence of the small nanoholes the structure has spatial-inversion-symmetry protected Dirac cones. In order to gap out these Dirac cones, additional small nanoholes are introduced in the unit cell to break the spatial inversion symmetry and reduce the system symmetry from $C_{6 v}$ down to $C_{3 v}$.

Since the unit cell of the $D 1$ domain is a mirror symmetric counterpart of that of the $D 2$ domain with respect to the domain-wall interface, the band diagram of the $D 1$ crystal is identical to that of the $D 2$ crystal. This key feature is important in our design, as it is particularly useful that the $D 1$ and $D 2$ crystals have a common bandgap. Moreover, since in the real space the $D 1$ domain is a mirror symmetric counterpart of the D2 domain, in momentum space the first Brillouin zone of the $D 1$ domain can be obtained from that of the $D 2$ domain by a rotation of 180 degrees, namely the $K$-valley points of the $D 1$ domain correspond to the $K^{\prime}$-valley points



Fig. 1: Schematic of a topological valley-Hall waveguide supporting valley-contrast unidirectional transport of terahertz plasmonic waves, i.e., RCP modes at the $K$ valley points and LCP modes at the $K^{\prime}$ valley points. The waveguide consists of a domain-wall interface constructed by placing together two mirror-symmetric graphene nanohole plasmonic crystals (domains $D 1$ and $D 2$ ), whose unit cells are highlighted by blue and red hexagons, respectively.

of the $D 2$ domain. As we will see later on, the valley mode at $K$ points is right-hand circularly polarized (RCP), whereas the valley mode at $K^{\prime}$ points is left-hand circularly polarized (LCP). This indicates that the valley states are inherently chiral, i.e., the chirality of the $K$-valley is opposite to that of the $K^{\prime}$-valley. Due to this important feature, a chiralitymomentum locked edge mode will emerge in the topological bandgap. In the following, we will discuss and analyze these ideas in more detail through full-model calculations of the band diagrams and propagation properties of the topological interfacial waveguide mode.

\section{RESUlTS AND DisCUSSION}

In this section we present the main results of our study. In particular, we first discuss the band structure of the graphene plasmonic crystals used in our work, as well as the mechanism through which an interfacial topological plasmonic crystal waveguide can be formed and its specific waveguiding properties.

\section{A. Band diagrams of graphene nanohole crystals}

As shown in Fig. 2(a), the unit cell of the proposed graphene plasmonic crystal contains two nanoholes with differen size. Moreover, the holes are arranged periodically in a hexagonal lattice, whose first Brillouin zone (FBZ) is depicted in Fig. 2(b). In this study, the lattice constant and the radius of the large holes are fixed as $a=400 \sqrt{3} \mathrm{~nm}$ and $R=140 \mathrm{~nm}$, respectively.

The optical properties of graphene are primarily characterized by its surface conductivity, which is generally given by the Kubo's formula [33], [34]. Within the random-phase approximation [35], [36], this formula can be expressed as the sum of inter-band and intra-band contributions, $\sigma_{s}=$ $\sigma_{\text {intra }}\left(\omega, \mu_{c}, \tau, T\right)+\sigma_{\text {inter }}\left(\omega, \mu_{c}, \tau, T\right)$. To be more specific, the intra-band part is given by:

$$
\sigma_{\text {intra }}=\frac{e^{2} k_{B} T \tau}{\pi \hbar^{2}(1-i \omega \tau)}\left[\frac{\mu_{c}}{k_{B} T}+2 \ln \left(e^{-\frac{\mu_{c}}{k_{B} T}}+1\right)\right],
$$

where $\mu_{c}$ is the chemical potential, $\tau$ is the relaxation time, $T$ is the temperature, $e$ is the electron charge, $k_{B}$ is the 
(a) Unit cell



(b) Brillouin zone

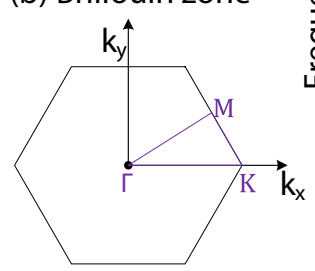

(c) 16

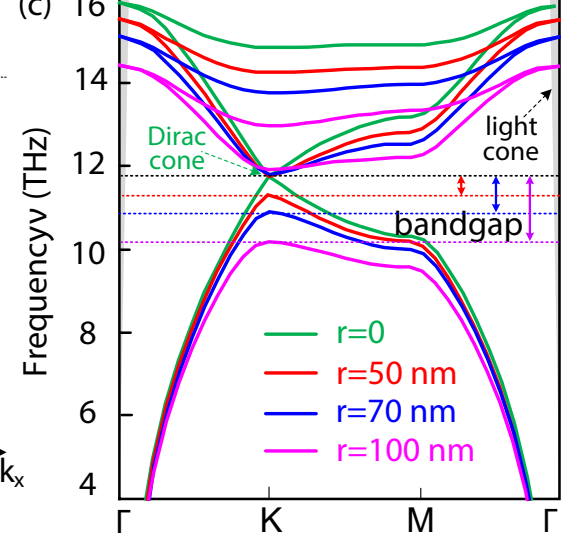

(a)

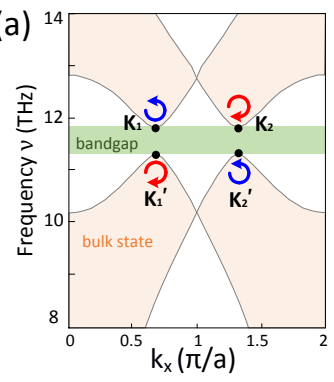

(b)

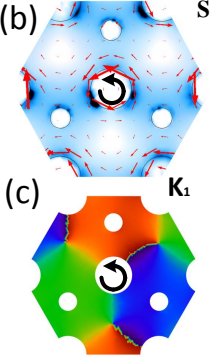

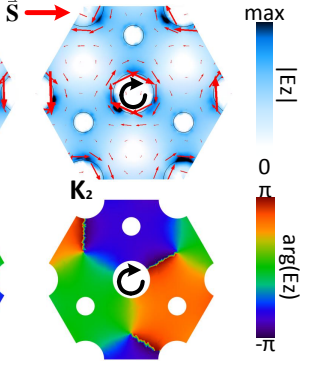

Fig. 3: Chirality properties of the bulk valley modes of graphene nanohole plasmonic crystals. (a) Projection of the band diagram of the graphene nanohole plasmonic crystal along the $\Gamma-K$ direction, where the four bulk valley modes at $K_{1}, K_{2}, K_{1}^{\prime}$ and $K_{2}^{\prime}$ are marked by dots. The chirality of the four valley modes are marked by clockwise- or counterclockwise-oriented arrows. (b) Electric field and Poynting vector distributions in a unit cell, determined in the $x-y$ plane for the $K_{1}$ and $K_{2}$ valley modes. A tight field confinement is suggested by the optical field distribution, whereas the distribution of the Poynting vector (red arrows) shows either a RCP or a LCP energy flow. (c) Phase distributions of the $E_{z}$ component in a unit cell, computed in the $x-y$ plane for the $K_{1}$ and $K_{2}$ valley modes, which show that the two valley modes have opposite chirality, i.e., for the $K_{1}$ and $K_{2}$ valley modes the $E_{z}$ phase vortex rotates either anticlockwise or clockwise around the center of the unit cell, respectively.

As a consequence, the corresponding topological plasmons can be formed at extremely deep subwavelength scale. In our case, the plasmon wavelength is around 40 times larger than the lattice constant.

In order to open up the inversion-symmetry-protected Dirac cone, small nanoholes are added to the plasmonic crystal, which means in this case $r \neq 0$. In this way, the symmetry point group of the unit cell shown in Fig. 2(a) is reduced from $C_{6 v}$ to $C_{3 v}$. Consequently, the $C_{6 v}$-symmetry-protected Dirac cone is gapped out, owing to the breaking of the spatial inversion symmetry. Moreover, the numerical results presented in Fig. 2(c) show that the width of the bandgap increases when the radius of the small nanoholes increases. To be more specific, the frequency bandwidth of the first bandgap increases from $1 \mathrm{THz}$ to about $2 \mathrm{THz}$ when the radius of the small nanoholes increases from $50 \mathrm{~nm}$ to $100 \mathrm{~nm}$.

The bulk valley modes obtained by gapping out the Dirac cone have nontrivial topological properties, which are responsible for the emergence of valley-Hall like topological domainwall interface modes to be discussed in the next subsection. To illustrate this phenomenon, we present in Fig. 3(a) the projected band diagram of the graphene nanohole plasmonic crystal. In this figure, the beige shaded regions represent the projection of the bulk modes, and the bandgap is marked by a green shaded region. The four bulk valley modes are labeled $K_{1}, K_{2}, K_{1}^{\prime}$, and $K_{2}^{\prime}$, where each valley mode has an intrinsic vorticity with the corresponding chirality marked by either a clockwise- or counterclockwise-oriented arrow. In practical applications, these optical vortices can be used to selectively 
excite different valley modes by choosing the specific angular momentum of the excitation source. To be more specific, if we input light with the frequency equal to the frequency of the upper valley modes, only RCP light could couple efficiently to the valley mode at $K_{1}$, whereas only LCP light couples to the valley mode at $K_{2}$.

In order to demonstrate this valley-chirality-locking property, the electric field and Poynting vector distributions of $K_{1}$ and $K_{2}$ valley modes are depicted in Fig. 3(b), where the colormap denotes the amplitude of $E_{z}$ component and the Poynting vector is depicted by red arrows (note that the $K_{1}^{\prime}$ and $K_{2}^{\prime}$ valley modes show similar behaviors, thus not shown). It can be seen in this figure that the electric field is tightly confined around the large nanoholes, thus illustrating the plasmon-induced near-field confinement effect. Moreover, the distribution of the Poynting vector shows that the chirality of the valley modes at $K_{1}$ and $K_{2}$ are RCP and LCP, respectively. Furthermore, this valley-chirality-locking feature also manifests through the corresponding distribution of the phase of $E_{z}$, as depicted in Fig. 3(c). To be more specific, the phase distribution of $E_{z}$ component at the $K_{1}$ valley winds counterclockwise from $\pi$ to $-\pi$ with respect to the center of the unit cell. On the contrary, the phase of $E_{z}$ at the $K_{2}$ valley point winds clockwise. Relying on this valley-chirality locking property, a valley-Hall topological graphene nanohole plasmonic crystal waveguide will be designed and studied in the following subsections.

\section{B. Band diagrams of graphene plasmonic crystal waveguides}

So far, we have explained how one can design a graphene nanohole plasmonic crystal that possesses a relatively wide bandgap. In order to construct valley-Hall topological edge modes inside this bandgap, we place together two optimized graphene nanohole plasmonic crystals to form a topological domain-wall interface, as illustrated in Fig. 4(a) and Fig. 4(b). In our study, the $D 2$ domain is a mirror symmetric counterpart of the $D 1$ domain. There are two key reasons why one constructs a valley-Hall topological domain-wall interface in this way.

First, apart from a symmetry transformation, the unit cell of the $D 1$ domain is identical to that of the $D 2$ domain. Therefore, one can avoid bandgap mismatch issues, namely the bandgaps of the plasmonic crystals in the domains $D 1$ and $D 2$ around the interface are perfectly matched. Second, in order to achieve a chirality-momentum locking feature of the edge modes supported by the domain-wall interface, the chirality of the $D 1$ domain should be opposite to that of the $D 2$ domain. This can be achieved by simply rotating in the momentum space by 180 degrees the unit cell of the $D 2$ domain or, equivalently, by ensuring that in the real space the domains $D 1$ and $D 2$ can be obtained from each other through a mirror symmetry transformation with respect to the interface. In other words, the $K$-valley points of the $D 1$ domain overlap with the $K^{\prime}$-valley points of the $D 2$ domain. Owing to this unique feature, the chirality of the modes in the nontrivial bandgap of the $D 1$ domain is opposite to that of the modes in the (same) nontrivial bandgap of the $D 2$ domain.
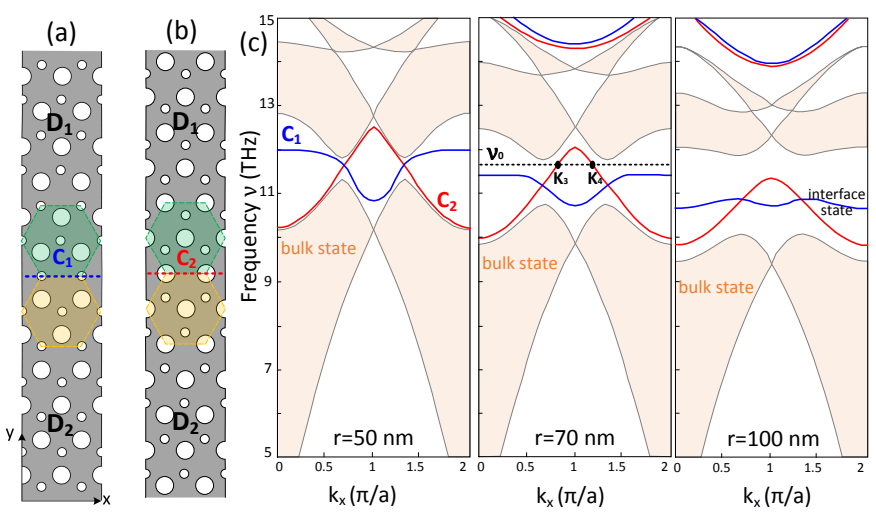

Fig. 4: Topological edge states of graphene nanohole plasmonic crystal waveguides. (a), (b) Two complementary domainwall interfaces $C_{1}$ (blue dashed line) and $C_{2}$ (red dashed line), respectively, which are constructed by placing together two mirror-symmetric domains, $D 1$ and $D 2$. (c) Band diagrams of graphene nanohole plasmonic crystal waveguides, determined for $r=50 \mathrm{~nm}, 70 \mathrm{~nm}$ and $100 \mathrm{~nm}$. Due to the presence of the domain-wall interfaces, two edge-mode bands are formed inside the topological bandgap. The blue (red) solid line corresponds to the edge-mode band of the domain-wall interface $C_{1}\left(C_{2}\right)$.

Since the domain-wall interface is constructed by placing together the $D 1$ and $D 2$ domains, it possesses a pair of counter-propagating edge modes with opposite chirality. Here, we should note that the domain-wall interface $C_{1}$ in Fig. 4(a) is a complementary counterpart of the domain-wall interface $C_{2}$ presented in Fig. 4(b), namely the two configurations can be obtained from each other by interchanging the positions of the large and small nanoholes. The structures depicted in Fig. 4(a) and Fig. 4(b), are periodic along the $x$-axis but are finite along the $y$-axis and symmetric with respect to the interface. Therefore, the topological features of the domainwall interface $C_{1}$ are complementary with respect to those of the domain-wall interface $C_{2}$. To simplify the discussion, in the following we only focus on the topological properties of the domain-wall interface $C_{2}$.

Using the FEM solver in Comsol Multiphysics 5.4, the band diagrams of the graphene nanohole plasmonic crystal waveguides depicted in Fig. 4(a) and Fig. 4(b) were calculated, and the corresponding results are given in Fig. 4(c). Due to the presence of the domain-wall interfaces, two additional valley-Hall topological edge-mode bands are formed inside the first bandgap of the graphene nanohole plasmonic crystal. Different from the topological edge-mode bands induced by the breaking of the time-reversal symmetry, these valley-Hall topological edge-state bands induced by the breaking of the spatial-inversion symmetry do not connect the conduction and valence bulk bands of the plasmonic crystal. In Fig. 4(c), the blue (red) solid line corresponds to the edge-state band of the domain-wall interface $C_{1}\left(C_{2}\right)$, whereas the beige shaded regions indicate the projection of the bulk modes. Furthermore, these band diagrams also suggest that the absolute value of the group velocity of the edge-state bands inside the topological 
bandgap decreases when the radius $r$ increases.

As it is well-known, as a consequence of slow-light effects, the smaller the absolute value of the group velocity is, the less confined the optical field will be at the domain-wall interface. Therefore, in order to avoid slow-light effects and the narrow operational bandwidth associated with flatten bands, the radius $r$ should be as small as possible. However, in most practical applications, it is desirable to use nanoholes with radius $r$ as large as possible, as this would make the fabrication processes less challenging and would also lead to a wider bandgap. After a careful consideration of both these factors, we found out that the optimum value of the radius $r$ was $70 \mathrm{~nm}$. Therefore, in the following analysis we used this value for $r$.

More specifically, when $r=70 \mathrm{~nm}$, the edge-state mode (red solid line) has a relatively large group velocity and is located in a topological bandgap with a rather large width of $2 \mathrm{THz}$. As illustrated in Fig. 4(c), if one excites this properly designed graphene nanohole plasmonic crystal waveguide using a monochromatic light source with frequency $\nu_{0}$, one can generate two distinct waveguide modes corresponding to the $K_{3}$ and $K_{4}$ points. More importantly, the group velocity of the edge-mode band at the $K_{3}$ point is positive, whereas the corresponding group velocity at the $K_{4}$ point is negative. As the propagation direction of a waveguide mode is determined by the sign of the corresponding group velocity, the guided waves corresponding to the $K_{3}$ and $K_{4}$ points propagate in opposite directions.

Importantly, as shown in Fig. 3(a), the chirality of the upper valley sate at the $K_{1}$ point has opposite value as compared to the chirality of the upper valley sate at the $K_{2}$ point. Consequently, the valley-Hall topological edge-mode at the $K_{3}$ and $K_{4}$ points have opposite chirality. A consequence of these properties of the chirality and group velocity at the $K_{3}$ and $K_{4}$ points is the unidirectional-propagation property of the topological waveguide modes of our graphene nanohole plasmonic crystal waveguide.

\section{Topological features of the plasmon propagation}

In order to further investigate the topological features of plasmon propagation in the proposed graphene nanohole plasmonic crystal waveguide, we considered the propagation properties of several graphene plasmonic crystal waveguide modes. First, in order to confirm the topologically-protected unidirectional propagation feature of the waveguide modes, a straight graphene plasmonic crystal waveguide is studied, as per Fig. 5(a). As shown in the left panel of this figure, a source located at the middle of the graphene plasmonic crystal waveguide generates right-circularly polarized monochromatic light with frequency of $\nu_{0}=11.5 \mathrm{THz}$. In our study, a source that emits circularly polarized light is implemented by placing at the corners of a hexagon six electric dipoles, which are marked by red dots in the inset of Fig. 5(a). The phase difference between neighboring electric dipoles is set to be $\pm \pi / 3$ so as to selectively excite a RCP or LCP phase vortex, respectively.

The chirality of the topological waveguide mode at the $K_{3}$ $\left(K_{4}\right)$ point is LCP $(\mathrm{RCP})$. Consequently, only the topological

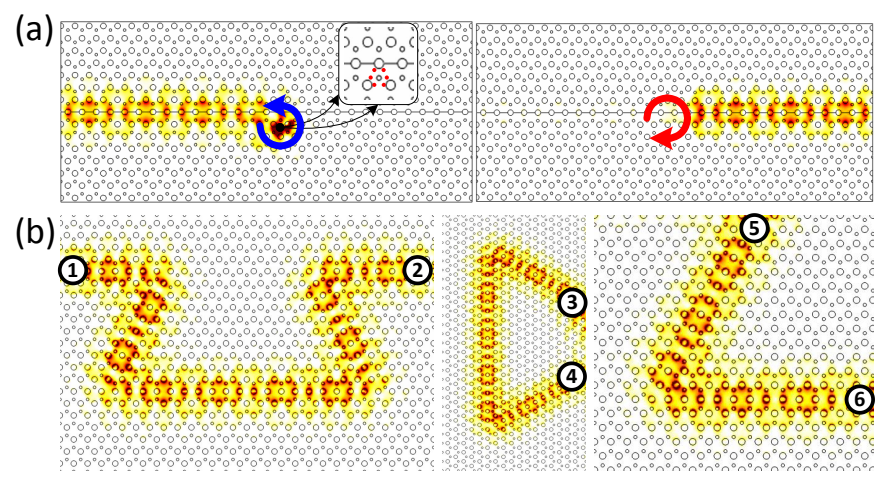

Fig. 5: Topological features of the topological waveguide mode propagation, including the unidirectional and the backscattering-immune propagation, in graphene nanohole plasmonic crystal waveguides. (a) Unidirectional propagation along the $-x$ direction (left panel) and a unidirectional propagation along the $+x$ direction (right panel) are achieved by placing in the topological waveguide optical sources that generate RCP light and a LCP light, respectively, at the frequency of $\nu_{0}=11.5 \mathrm{THz}$. (b) Bend-immune propagation in three distinct sharply curved domain-wall interfaces: $U$ bend in the left panel, $C$ bend in the middle panel, and $L$ bend in the right panel. Backscattering-immune propagation is observed, meaning that the proposed graphene nanohole plasmonic crystal waveguide modes are robust against bend perturbations.

waveguide mode at the $K_{4}$ point could be excited in the left panel of Fig. 5(a), if we use as excitation a RCP light source. Moreover, since the group velocity of the topological waveguide mode at the $K_{4}$ point is negative in Fig. 4(c), the generated optical field will propagate along the $-x$ direction. As a consequence of these two features, a unidirectional propagation of plasmonic waves is achieved, which is illustrated in the left panel of Fig. 5(a). Similarly, as demonstrated in the right panel of Fig. 5(a), unidirectional propagation along $+x$ direction of plasmonic waves with LCP chirality can be achieved in this graphene nanohole plasmonic crystal waveguide if one uses as excitation a source that generates left-circularly polarized light.

In addition to the unidirectional propagation, the topological feature of backscattering-immune propagation in the proposed graphene nanohole plasmonic crystal waveguide is also validated. As illustrated in Fig. 5(b), three sharply curved domainwall interfaces with different shapes ( $U$ bend in the left panel, $C$ bend in the middle panel, and $L$ bend in the right panel) are used to test the backscattering-immune propagation in the proposed graphene nanohole plasmonic crystal waveguides. In the case of the $U$ bend, monochromatic light with frequency $\nu_{0}$ is launched at the waveguide port (1), whereas the transmitted power is collected at the waveguide port (2). The corresponding near-field distribution in the left panel of Fig. 5(b) shows that the light propagation is topologically-protected, as it is immune to several sharp-bend corners. This feature is particularly promising for the design of photonic time-delay lines. Similar backscattering-immune propagation can also be 


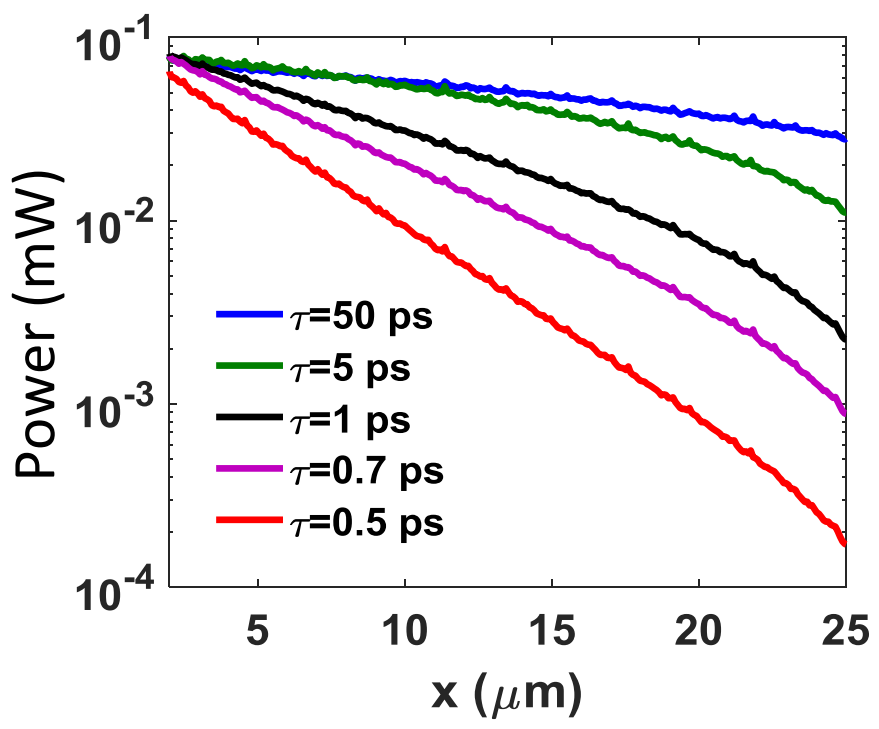

Fig. 6: Dependence of the modal power on the propagation distance, determined for different values of the lifetime of the graphene plasmon.

observed in the $C$ and $L$ bends. In the case of the $C$ bend, the feeding waveguide port (3) and the output waveguide port (4) are placed at the same side of the crystal. Due to the topological protection, a backscattering-immune propagation is achieved in this case, too. This remarkable feature can be used in the design of a photonic device that reverses the direction of light propagation. In the case of the $L$ bend, the feeding waveguide port (5) is placed at the top side of the crystal, whereas the output waveguide port (6) is located at its right side. Similarly to the phenomena observed in the cases of the $U$ and $C$ bends, in this case, too, the backscattering of light is efficiently suppressed. This property can be used to the design of a photonic lateral diverter.

\section{The effect of graphene loss on the topological light prop- agation}

As shown in Fig. 2(c), the first bandgap of the graphene nanohole plasmonic crystals is located at frequencies that are far below the light cone. Thus, the valley-Hall topological edge modes inside this bandgap are guided modes, as depicted in Fig. 4(c), and therefore the radiation losses vanish. Moreover, the plasmonic field is tightly confined at the domain-wall interface, which can lead to increased optical losses due to the intrinsic loss of graphene. Typically, the lifetime of graphene plasmons due to plasmon-phonon scattering varies from $0.1 \mathrm{ps}$ to $1 \mathrm{ps}$ [37]. However, recently it has been demonstrated that the lifetime of graphene plasmons can be increased to about 3 ps if the exfoliated graphene is placed onto a boron nitride substrate [38]. Moreover, the lifetime of graphene plasmons under a strong static magnetic field can be as large as $50 \mathrm{ps}$, as demonstrated in [39], [40].

Guided by these experimental considerations, we have investigated the effect of the intrinsic loss of graphene on the characteristics of the valley-Hall topological plasmon propagation in a graphene nanohole plasmonic crystal waveguide.

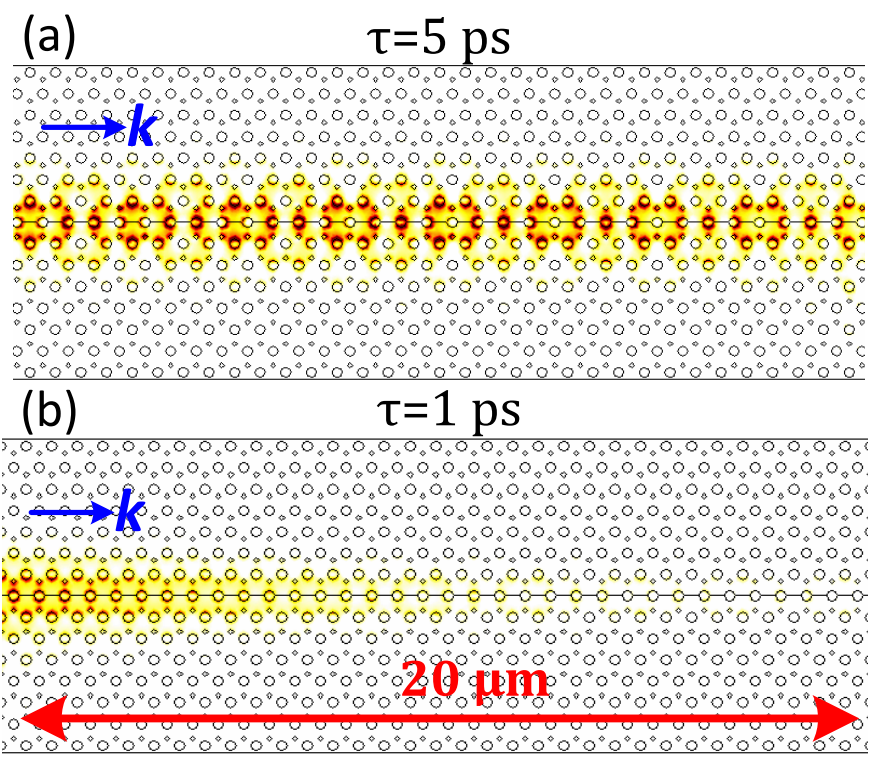

Fig. 7: (a), (b) Spatial distribution of the electric field intensity, $|E|$, determined for a lifetime of graphene plasmons of $\tau=$ $5 \mathrm{ps}$ and $\tau=1 \mathrm{ps}$, respectively.

As shown in Fig. 6, the dependence of the power of the topological waveguide modes on the propagation distance is evaluated via a surface integral of the Poynting vector over a cross section in the $y-z$ plane. Owing to the intrinsic loss of graphene, as expected, the optical power of the waveguide mode decreases as the propagation distance increases. The power decay rate can be quantified by the characteristic loss length, $L_{\text {loss }}$, defined as $L_{\text {loss }}=1 / \alpha$, where the loss coefficient $\alpha$ is defined by the relation:

$$
P(x)=P(0) e^{-\alpha x} .
$$

For our topological waveguide mode, $L_{\text {loss }}=27 \mu \mathrm{m}, 20 \mu \mathrm{m}$, $8.3 \mu \mathrm{m}, 5.9 \mu \mathrm{m}$ and $4 \mu \mathrm{m}$ when $\tau=50 \mathrm{ps}, 5 \mathrm{ps}, 1 \mathrm{ps}, 0.7 \mathrm{ps}$ and $0.5 \mathrm{ps}$, respectively.

A further illustration of the influence of the intrinsic loss of graphene on the plasmon propagation in the proposed graphene plasmonic crystal waveguide is provided by the electric field distributions presented in Fig. 7. In both cases the propagation length was equal to $20 \mu \mathrm{m}$, whereas the lifetime of the graphene plasmons was $\tau=5 \mathrm{ps}$ in Fig. 7(a) and $\tau=1 \mathrm{ps}$ in Fig. 7(b). The results of these numerical simulations clearly illustrate the dramatic effect that the intrinsic graphene losses has on the characteristic propagation length of the topological waveguide modes investigated in this work.

\section{CONCLUSION}

In summary, we have proposed for the first time a novel valley-Hall topological plasmonic crystal waveguide formed at the interface between two mirror-symmetric graphene crystals placed in close contact. After an optimization of the structure of the crystals, a wide topological bandgap containing an interfacial topological waveguide mode is achieved. Using full-model simulations, the band diagram of this graphene plasmonic crystal has been investigated, and a topological 
edge band was observed inside the bandgap. More importantly, we show that this topological edge band can exist at extremely deep-subwavelength scale, namely for $\lambda / a>40$. Moreover, we demonstrate unidirectional and backscatteringimmune propagation of the proposed graphene plasmonic crystal waveguide modes, key features that highlight their topological nature. In addition, the effect of the intrinsic loss of graphene on the valley-Hall topological propagation in the proposed graphene nanohole plasmonic crystal waveguide has been investigated, too. We found that characteristic loss lengths of tens of micrometers can be achieved provided that the plasmon relaxation time is $\gtrsim 1$ ps. These important properties of valley-Hall topological plasmons could play an important rôle in the development of highly-integrated and robust photonic crystal waveguides at deep-subwavelength scale.

\section{ACKNOWLEDGMENTS}

The authors acknowledge the use of the UCL Legion High Performance Computing Facility (Legion@UCL), and associated support services, in the completion of this work.

\section{REFERENCES}

[1] T. Ozawa et al., "Topological photonics," Rev. Mod. Phys., vol. 91, no. 1, pp. 015006, Mar. 2019.

[2] F. D. M. Haldane and S. Raghu, "Possible realization of directional optical waveguides in photonic crystals with broken time-reversal symmetry," Phys. Rev. Lett., vol. 100, no. 1, pp. 013904, Jan. 2008.

[3] Z. Wang, Y. Chong, J. D. Joannopoulos, and M. Soljacic, "Observation of unidirectional backscattering-immune topological electromagnetic states," Nature, vol. 461, no. 7265, pp. 772-775, Oct. 2009.

[4] L. Lu, J. D. Joannopoulos, and M. Soljacic, "Topological photonics," Nat. Photonics, vol. 8, no. 11, pp. 821-829, Oct. 2014.

[5] L. Lu, J. D. Joannopoulos, and M. Soljacic, "Topological states in photonic systems," Nat. Phys., vol. 12, no. 7, pp. 626-629, June 2016.

[6] A. B. Khanikaev and G. Shvets, "Two-dimensional topological photonics," Nat. Photonics, vol. 11, no. 12, pp. 763-773, Nov. 2017.

[7] C. Liu, W. Gao, B. Yang, and S. Zhang, "Disorder-induced topological state transition in photonic metamaterials," Phys. Rev. Lett., vol. 119, no. 18, pp. 183901, Nov. 2017.

[8] Z. Wang, Y. D. Chong, J. D. Joannopoulos, and M. Soljacic, "Reflectionfree one-way edge modes in a gyromagnetic photonic crystal," Phys. Rev. Lett., vol. 100, no. 1, pp. 013905, Jan. 2008.

[9] Y. Yang, Y. Poo, R. X. Wu, Y. Gu, and P. Chen, "Experimental demonstration of one-way slow wave in waveguide involving gyromagnetic photonic crystals," Appl. Phys. Lett., vol. 102, no. 23, pp. 231113, May 2013.

[10] S. Mittal et al., "Topologically robust transport of photons in a synthetic gauge field," Phys. Rev. Lett., vol. 113, no. 8, pp. 087403, Aug. 2014.

[11] M. Hafezi, S. Mittal, J. Fan, A. Migdall, and J. M. Taylor, "Imaging topological edge states in silicon photonics," Nat. Photon., vol. 7, no. 12, pp. 1001-1005, Oct. 2013.

[12] X. Cheng et al., "Robust reconfigurable electromagnetic pathways within a photonic topological insulator," Nat. Mater, vol. 15, no. 5, pp. 542548, Feb. 2016.

[13] B. Bahari et al., "Nonreciprocal lasing in topological cavities of arbitrary geometries," Science, vol. 358, no. 6363, pp. 636-640, Nov. 2017.

[14] P. St-Jean et al., "Lasing in topological edge states of a one-dimensional lattice," Nat. Photon., vol. 11, no. 10, pp. 651-656, Sep. 2017.

[15] K. S. Novoselov et al., "Electric field effect in atomically thin carbon films," Science, vol. 306, no. 5696, pp. 666-669, Oct. 2004.

[16] K. S. Novoselov et al., "Two-dimensional gas of massless Dirac fermions in graphene," Nature, vol. 438, no. 7065, pp. 197-200, Nov. 2005.

[17] A. N. Grigorenko, M. Polini, and K. S. Novoselov, "Graphene plasmonics," Nat. Photonics, vol. 6, no. 11, pp. 749-758, Nov. 2012.

[18] P. Avouris and M. Freitag, "Graphene photonics, plasmonics, and optoelectronics," IEEE J. Sel. Top. Quantum Electron., vol. 20, no. 1, pp. 72-83, Jan. 2014.
[19] A. Tredicucci and M. S. Vitiello, "Device concepts for graphene-based terahertz photonics," IEEE J. Sel. Top. Quantum Electron., vol. 20, no. 1, pp. 130-138, Jan. 2014.

[20] A. Woessner et al., "Highly confined low-loss plasmons in grapheneboron nitride heterostructures," Nat. Mater., vol. 14, no. 4, pp. 421-425, Jan. 2015.

[21] B. Yao et al., "Broadband gate-tunable terahertz plasmons in graphene heterostructures," Nat. Photonics, vol. 12, no. 1, pp. 22-28, Jan. 2018.

[22] G. X. Ni et al., "Fundamental limits to graphene plasmonics," Nature, vol. 557, no. 7706, pp. 530-533, May 2018.

[23] D. Jin et al., "Infrared topological plasmons in graphene," Phys. Rev. Lett., vol. 118, no. 24, pp. 245301, June 2017.

[24] D. Pan, R. Yu, H. Xu, and F. J. G. de Abajo, "Topologically protected Dirac plasmons in a graphene superlattice," Nat. Commun., vol. 8, no. 1, pp. 1243, Nov. 2017

[25] J. W. You, Z. Lan, and N. C. Panoiu, "Four-wave Mixing of Topological Edge Plasmons in Graphene Metasurfaces," Sci. Adv, vol. 6, no. 13, art. no. eaaz3910, Mar. 2020.

[26] M. Jung, Z. Fan, and G. Shvets, "Midinfrared plasmonic valleytronics in metagate-tuned graphene," Phys. Rev. Lett., vol. 121, no. 8, pp. 086807, Aug. 2018.

[27] P. Qiu et al., "Topologically protected edge states in graphene plasmonic crystals," Opt. Express, vol. 25, no. 19, pp. 22587-22594, Sep. 2017.

[28] P. Qiu et al., "Plasmonic valley chiral states in graphene based plasmonic crystals," J. Phys. D: Appl. Phys., vol. 52, no. 1, pp. 015102, Oct. 2018.

[29] A. Sinitskii and J. M. Tour, "Patterning graphene through the selfassembled templates: toward periodic two-dimensional graphene nanostructures with semiconductor properties," J. Am. Chem. Soc., vol.132, no.42, pp.14730-14732, Oct. 2010.

[30] J. Yang et al., "Graphene nanomesh: new versatile materials," Nanoscale, vol.6, no.22, pp.13301-13313, Sep. 2014

[31] K. K. Gopalan et al., "Scalable and tunable periodic graphene nanohole arrays for mid-infrared plasmonics," Nano Lett., vol. 18, no. 9, pp. 59135918, Aug. 2018

[32] K. Y. M. Yeung et al., "Far-infrared graphene plasmonic crystals for plasmonic band engineering," Nano Lett., vol. 14, no. 5, pp. 2479-2484, Apr. 2014

[33] P. A. D. Goncalves and N. M. Peres, An Introduction to Graphene Plasmonics (World Scientific, 2016)

[34] J. W. You, S. R. Bongu, Q. Bao, and N. C. Panoiu, "Nonlinear optical properties and applications of 2D materials: theoretical and experimental aspects," Nanophotonics, vol. 8, no.1, pp. 63-97, Dec. 2018.

[35] G. W. Hanson, "Dyadic Green's functions and guided surface waves for a surface conductivity model of graphene," J. Appl. Phys., vol. 103, no. 6, pp. 064302, Mar. 2008.

[36] D. W. Wang, W. S. Zhao, X. Q. Gu, W. C. Chen, and W. Y. Yin, "Wideband modeling of graphene-based on structures at different temperatures using hybrid FDTD method," IEEE Trans. Nanotechnol., vol. 14, no. 2, pp. 250-258, Jan. 2015.

[37] T. Low and P. Avouris, "Graphene plasmonics for terahertz to midinfrared applications," ACS Nano, vol. 8, no. 2, pp. 1086-1101, Jan. 2014.

[38] C. R. Dean et al., "Boron nitride substrates for high-quality graphene electronics," Nat. Nanotechnol., vol. 5, no. 10, pp. 722-726, Aug. 2010.

[39] H. Yan et al., "Infrared spectroscopy of tunable Dirac terahertz magnetoplasmons in graphene," Nano Lett., vol. 12, no. 7, pp. 3766-3771, June 2012.

[40] I. Petkovic et al., "Carrier drift velocity and edge magnetoplasmons in graphene," Phys. Rev. Lett., vol. 110, no. 1, pp. 016801, Jan. 2013.

[41] Comsol multiphysics, COMSOL Multiphysics Modeling Software, 2020, https://uk.comsol.com/.

[42] A. Slobozhanyuk et al., "Three-dimensional all-dielectric photonic topological insulator," Nat. Photonics, vol. 11, no. 2, pp. 130-136, Feb. 2017.

[43] X. T. He et al., "A silicon-on-insulator slab for topological valley transport," Nat. Commun., vol. 10, no. 1, pp. 872, Feb. 2019. 


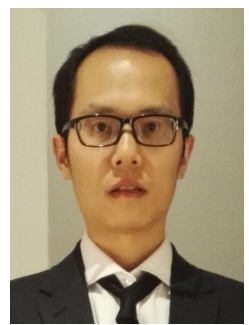

Jian Wei You received the B.Sc. degree in electrical engineering from Xidian University, Xi'an, China, in 2010, and the Ph.D. degree in electromagnetic and microwave technology from Southeast University, Nanjing, China, in 2016. From July 2011 to December 2011, he served as a Research Visitor at the Department of Electrical and Computer Engineering, University of Houston, Houston, USA. In January 2016, he joined the Department of Electronic and Electrical Engineering, University College London, as a Research Associate.

His research interests include computational electromagnetics, nonlinear microwave and optics, quantum metamaterials, multi-physics, and computational plasma physics. Dr. You is a member of the IEEE.

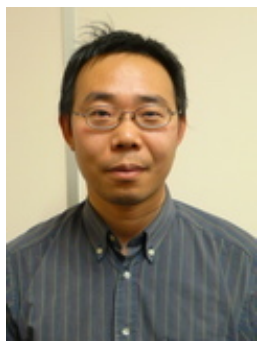

Zhihao Lan received the B.Sc. and M.Sc. degrees in physics from the Beijing Institute of Technology, and Peking University, Beijing, China in 2004 and 2007, respectively, and the Ph.D. degree in physic$\mathrm{s}$ from Heriot-Watt University, Edinburgh, UK in 2012. He worked as postdoctoral research fellow in Southampton University, UK, from 2011 to 2014 and Nottingham University, UK, from 2014 to 2017 . He joined the Department of Electronic and Electrical Engineering, University College London, in 2018 as a research associate in theoretical and computational modeling of quantum photonic materials and devices.

His research interests include quantum optics, quantum gases, quantum many-body physics, quantum non-equilibrium systems, novel quantum systems for quantum simulations and quantum technologies, topological states of matter, and topological photonics and plasmonics.

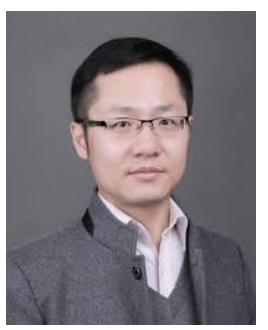

Qiaoliang Bao received his B. A. (2000) and M. E. (2003) degrees in Materials Science and Engineering from Wuhan University of Technology (China). He obtained his Ph.D. degree (2007) in Materials Physics and Chemistry from Wuhan University (China). He worked as postdoctoral fellow at Nanyang Technological University and National University of Singapore from 2007 to 2012. He was appointed as a tenured associate professor at Department of Materials Science and Engineering, Monash University in 2016.

His research interests include waveguide-coupled 2D semiconductors and polariton-coupled 2D materials and devices, focusing on the effect of confinedspace light-matter interactions on the transport of electrons or other quasiparticles such as plasmon polariton, exciton polarition and phonon polariton.

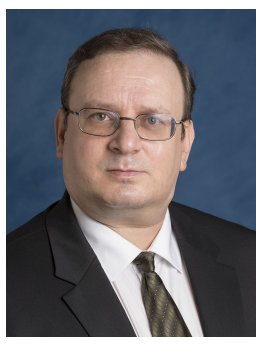

Nicolae C. Panoiu received the B.Sc. and M.S degrees in physics from the University of Bucharest, Bucharest, Romania, in 1990 and 1992, respectively, and the Ph.D. degree from New York University, New York, in 2001. After graduating from NYU, he was a Postdoctoral Fellow in the Department of Applied Physics and Applied Mathematics, Columbia University, New York. He is currently Professor of Nanophotonics in the Department of Electronic and Electrical Engineering, University College London.

His research interests include silicon photonics, optical properties of photonic nanostructures and metamaterials, and computational modeling of electromagnetic structures. Prof. Panoiu is the recipient of an ERC Consolidator Grant and is a member of the IEEE and OSA. 